\title{
The expected Shapley value
}

Citation for published version (APA):

Maus, S. (2003). The expected Shapley value. METEOR, Maastricht University School of Business and Economics. METEOR Research Memorandum No. 004 https://doi.org/10.26481/umamet.2003004

Document status and date:

Published: 01/01/2003

DOI:

10.26481/umamet.2003004

Document Version:

Publisher's PDF, also known as Version of record

\section{Please check the document version of this publication:}

- A submitted manuscript is the version of the article upon submission and before peer-review. There can be important differences between the submitted version and the official published version of record.

People interested in the research are advised to contact the author for the final version of the publication, or visit the DOI to the publisher's website.

- The final author version and the galley proof are versions of the publication after peer review.

- The final published version features the final layout of the paper including the volume, issue and page numbers.

Link to publication

\footnotetext{
General rights rights.

- You may freely distribute the URL identifying the publication in the public portal. please follow below link for the End User Agreement:

www.umlib.nl/taverne-license

Take down policy

If you believe that this document breaches copyright please contact us at:

repository@maastrichtuniversity.nl

providing details and we will investigate your claim.
}

Copyright and moral rights for the publications made accessible in the public portal are retained by the authors and/or other copyright owners and it is a condition of accessing publications that users recognise and abide by the legal requirements associated with these

- Users may download and print one copy of any publication from the public portal for the purpose of private study or research.

- You may not further distribute the material or use it for any profit-making activity or commercial gain

If the publication is distributed under the terms of Article $25 \mathrm{fa}$ of the Dutch Copyright Act, indicated by the "Taverne" license above, 


\title{
The expected Shapley value ${ }^{1}$
}

\author{
Stefan Maus \\ Date of this version: February 10, 2003 \\ Department of Quantitative Economics, University of Maastricht, P.O. Box 616, \\ 6200 MD Maastricht, THE NETHERLANDS (email: s.maus@ke.unimaas.nl)
}

Summary. A method to allocate the benefits to the players of a cooperative game is the Shapley value. Its computation demands the knowledge of all coalition worths with certainty. This paper introduces the expected Shapley value, an extension of the Shapley value to games where not all the worths are known with certainty. The expected Shapley value is characterized with adapted versions of Young's (1985) and Shapley's (1953) properties. It is shown how representation with unanimity games, dividend and potential generalize when not all worths are known with certainty. We relate the expected Shapley value to the reduced and the normalized Shapley value introduced by Willson (1993) and Housman (2001) for games where some coalition worths are not known.

Keywords and Phrases: value, cooperative game, axioms, partially defined games, belief.

\section{Introduction}

The Shapley value (Shapley, 1953) is a method to allocate benefits to the players of a cooperative game. However, the Shapley value requires a large amount of information, since it takes into account the worth of any coalition. As the number of coalitions grows exponentially with the number of players, one might want to consider cooperative games where only a part of these worths are known. Such games are called partially defined games, and the coalitions whose worths are known in that game are collected in a coalition list, which is a subset of the set of all coalitions.

\footnotetext{
${ }^{1}$ The author thanks Ton Storcken and Hans Peters for their helpful suggestions.
} 
Willson (1993) and Housman (2001) characterized extensions of the Shapley value to partially defined games with symmetric coalition lists: $b$-weighted Shapley value, reduced Shapley value and normalized Shapley value. These extensions are based on extensions of axioms of efficiency, anonymity, linearity and dummy-player. The original axioms characterize the Shapley value for cooperative games. Housman (2001), however, obtains several impossibility results, in terms of coalition lists admitting such extensions of the Shapley value. These impossibilities suggest that the axioms used are too strong, which is made more explicit by an example in section 2. In particular linearity is too strong, as stated already in the conclusion of Housman (2001). The reason is that any extension of the sum of two games can arise in multiple ways from extensions of the two original games, but for some extensions of the sum there are more such ways than for others.

The path we take in this paper differs somewhat from that of Willson (1993) and Housman (2001). We use their concept of a partially defined cooperative game with coalition list. Additionally we assume that players have a belief on the underlying cooperative games, where all worths are known. This belief could arise from players who believe that they are playing a game of a certain type, for example a superadditive game, and consider any underlying superadditive extension a priori equally likely. These beliefs in turn allow us to characterize the expected Shapley value. This value of a partially defined cooperative game can be argued to arise in the following way. First, given the belief of the players, compute the Shapley value of any of the games that could be the underlying cooperative game. Integrate these values with respect to the probability distribution given by the belief of the players. The resulting value is the expected Shapley value. The expected Shapley value encompasses the reduced and normalized Shapley value as special cases, i.e. for a certain type of belief. The approach used here also allows for any coalition list, possibly asymmetric, containing the empty coalition. The price we have to pay for the expected Shapley value is, that, compared to the reduced Shapley value and normalized Shapley value, more information is required since beliefs about the underlying games are needed. But this is still a weaker requirement than knowing all worths with certainty. A common assumption in practise might be to take uniform distributions over games with standard properties, such as convexity, superadditivity or monotonicity. 
In the next section the relevant definitions are given and an example of a nonlinear solution is discussed. Section 3 contains a characterization of the expected Shapley value with a standard set of properties à la Young (1985), of which actually only monotonicity has to be adapted to take into account beliefs. After that section 4 explores a weaker form of linearity, which is used in another characterization of the expected Shapley value, this time with a standard set of properties à la Shapley (1953). Then section 5 explains how games with beliefs can be represented by unanimity games, and how the concepts of dividend and potential extend. In section 6 we show how to obtain the reduced and the normalized Shapley value as expected Shapley values. Finally, section 7 concludes the paper.

\section{Partially defined cooperative games}

Throughout, when vectors are compared, this is done coordinatewise.

A set of players $N$ is a finite nonempty subset of the natural numbers $\mathbb{N}$. A subset $S$ of $N$ is called a coalition, and we write $|S|$ for the number of players in the coalitions. Next we introduce the class of partially defined cooperative games. Let $\mathcal{M} \subseteq 2^{N}, \emptyset \in \mathcal{M}$ be a subset of coalitions, then $\mathcal{M}$ is called a coalition list in $N$. A pair $(N, v)$ consisting of a function $v: \mathcal{M} \rightarrow \mathbb{R}$ such that $v(\emptyset)=0$ and a player set $N$ is called a partially defined cooperative game with coalition list $\mathcal{M}$. We will also call it an $\mathcal{M}$-game. We call $v(S)$ the worth of coalition $S$ if $S$ is in $\mathcal{M}$. If $S$ is not in $\mathcal{M}$ then the interpretation is that the worth of this coalition is not known. It might be that worths are costly to determine, for example. A coalition list $\mathcal{M}$ is symmetric, if for any $S \in \mathcal{M}$, any coalition of the same cardinality is also in $\mathcal{M}$. It is easily seen that if $\mathcal{M}$ is symmetric, then there is a set of known coalition sizes $K \subseteq\{0, \ldots,|N|\}$ such that

$$
\mathcal{M}=\mathcal{M}(K):=\{S \subseteq N|| S \mid=k \text { for some } k \in K\} .
$$

If there is no confusion the $\mathcal{M}$-game $(N, v)$ is referred to by $v$. Note that it is not necessary to define a partially defined cooperative game as a triple $(N, \mathcal{M}, v)$, since $\mathcal{M}=\operatorname{dom}(v)$ is given by the domain of $v .^{2}$

\footnotetext{
${ }^{2}$ Any function is also a relation, so $v \subseteq 2^{N} \times \mathbb{R}$ and the domain of $v$ is given by the projection on the first coordinate of $v$.
} 
The set of all partially defined games with player set $N$ is denoted by $P G^{N}$. So

$$
P G^{N}=\{v: \mathcal{M} \rightarrow \mathbb{R} \mid \mathcal{M} \text { is a coalition list in player set } N, v(\emptyset)=0\} .
$$

A cooperative game with player set $N$ is a partially defined cooperative game $(N, w)$, where $w$ has domain $\mathcal{M}=2^{N}$. Since then all coalition worths are known we will also refer to cooperative games as fully defined games. The set of all cooperative games with player set $N$ is denoted by $G^{N}$. A value solution on $G^{N}$ is a function $f: G^{N} \rightarrow \mathbb{R}^{N}$. The number $f_{i}(w)$ is interpreted as the share of player $i$ in the game $w \in G^{N}$. The Shapley value $S h: G^{N} \rightarrow \mathbb{R}^{N}$ for a cooperative game $w \in G^{N}$ is defined by

$$
S h_{i}(w):=\sum_{i \notin S, S \subseteq N} \frac{|S| !(|N|-1-|S|) !}{|N| !}(w(S \cup\{i\})-w(S)),
$$

for all $i \in N$. In this paper we aim to extend to Shapley value on $G^{N}$ to partially defined cooperative games. The set $G^{N}$ of fully defined games with player set $N$ is a subset of $P G^{N}$. The set of all fully defined games is given by

$$
G:=\bigcup\left\{G^{N}|\emptyset \neq N \subseteq \mathbb{N},| N \mid<\infty\right\} .
$$

Likewise, the set of all partially defined games is given by

$$
P G:=\bigcup\left\{P G^{N}|\emptyset \neq N \subseteq \mathbb{N},| N \mid<\infty\right\} .
$$

Again, $G$ is a subset of $P G$.

Let $(N, v)$ be a partially defined cooperative game with coalition list $\mathcal{M}$. Any fully defined game $w \in G^{N}$ satisfying $\left.w\right|_{\mathcal{M}}=v$ is called an extension of $v$.

Some important subspaces of the set of all fully defined games are the monotonic games

$$
M O^{N}:=\left\{w \in G^{N} \mid w(S) \leq w(T) \text { for all } S, T \in 2^{N} \text { with } S \subseteq T\right\},
$$

the superadditive games

$S A^{N}:=\left\{w \in G^{N} \mid w(S)+w(T) \leq w(S \cup T)\right.$ for all $S, T \in 2^{N}$ with $\left.S \cap T=\emptyset\right\}$

and the convex games

$$
C O^{N}:=\left\{w \in G^{N} \mid w(S)+w(T) \leq w(S \cup T)+w(S \cap T) \text { for all } S, T \in 2^{N}\right\} .
$$


We proceed with an example, where players believe that each game from a certain set of extensions to fully defined games has equal probability to be the underlying game. This gives rise to a nonlinear solution to the allocation problem. Thus the example shows that linearity cannot be adapted in a straightforward fashion. The reason is that when two games are added, any extension of the sum of these games can be expressed as a sum of extensions of the two games added, but some extensions are 'more likely' than others to arise in this way.

Example 1 There are three players $N=\{1,2,3\}$. The coalition list is

$$
\mathcal{M}=\{\varnothing,\{1\},\{2\},\{3\},\{1,2\}, N\} .
$$

So only the worths of the coalitions $\{1,3\}$ and $\{2,3\}$ are unknown. Let $v$ be the $\mathcal{M}$-game satisfying

$$
\begin{aligned}
v(N) & =1, \\
v(\{i\}) & =0 \text { for all } i \in N, \\
v(\{1,2\}) & =0 .
\end{aligned}
$$

Suppose that players believe that they are playing a convex game. The constraints for extensions $w$ derived from the convexity conditions are

$$
\begin{gathered}
w(\{1,3\})+w(\{2,3\}) \leq v(N)=1, \\
w(\{1,3\}) \geq 0 \text { and } w(\{2,3\}) \geq 0 .
\end{gathered}
$$

Hence, the set of convex extensions of $v$ can be identified with the set

$$
W:=\{(x, y) \mid x, y \geq 0 \text { and } x+y \leq 1\} .
$$

Let $\widehat{v}$ be an $\mathcal{M}$-game which equals $v$ except for $\widehat{v}(\{1,2\})=\frac{1}{2}$. Then for any convex extension $\widehat{w}$ we have that $0 \leq \widehat{w}(\{1,3\}) \leq \frac{1}{2}$ and $0 \leq \widehat{w}(\{2,3\}) \leq \frac{1}{2}$. This set of extensions can be identified with the set

$$
\widehat{W}:=\left[0, \frac{1}{2}\right]^{2} .
$$

The players consider each game equally likely so they might agree to compute the expected games $\mathbb{E}_{v}$ and $\mathbb{E}_{\widehat{v}}$ under the uniform distribution on $W$ and $\widehat{W}$, respectively, and distribute according to the Shapley value of these expected 
games. Let $\lambda^{2}$ be the two-dimensional Lebesgue measure on $\mathbb{R}^{2}$. As the area of $W$ is $\frac{1}{2}$, the density $f$ of the uniform distribution $F$ on $W$ with respect to $\lambda^{2}$ is given by

$$
f(x, y)=\left\{\begin{array}{cc}
2 & \text { if }(x, y) \in W \\
0 & \text { otherwise. }
\end{array}\right.
$$

For symmetry reasons we have $\mathbb{E}_{v}(\{1,3\})=\mathbb{E}_{v}(\{2,3\})$. We obtain, using the density $f$ and standard integration techniques,

$$
\begin{aligned}
\mathbb{E}_{v}(\{1,3\}) & =\int_{W} x d F(x, y) \\
& =\frac{1}{3} .
\end{aligned}
$$

For $\mathbb{E}_{\widehat{v}}$ we have obviously $\mathbb{E}_{\widehat{v}}(\{1,3\})=\mathbb{E}_{\widehat{v}}(\{2,3\})=\frac{1}{4}$. On the set of known coalitions $\mathcal{M}$ the expected games equal the partially defined games. It is easy to see that the set of convex extensions of the sum of the two games $v+\widehat{v}$ is the set $W+\widehat{W}$. Using that the area of $W+\widehat{W}$ is $\frac{7}{4}$, we obtain in the same way as for $\mathbb{E}_{v}$, that the expected game $\mathbb{E}_{v+\widehat{v}}$ will satisfy

$$
\begin{aligned}
\mathbb{E}_{v+\widehat{v}}(\{1,3\}) & =\mathbb{E}_{v+\widehat{v}}(\{2,3\}) \\
& =\frac{89}{84} .
\end{aligned}
$$

The Shapley values of the games $\mathbb{E}_{v}, \mathbb{E}_{\widehat{v}}$ and $\mathbb{E}_{v+\widehat{v}}$ are

$$
\begin{aligned}
S h\left(\mathbb{E}_{v}\right) & =\left(\frac{5}{18}, \frac{5}{18}, \frac{8}{18}\right) \\
S h\left(\mathbb{E}_{\widehat{v}}\right) & =\left(\frac{3}{8}, \frac{3}{8}, \frac{1}{4}\right) \text { and } \\
S h\left(\mathbb{E}_{v+\widehat{v}}\right) & =\left(\frac{289}{504}, \frac{289}{504}, \frac{215}{252}\right) .
\end{aligned}
$$

So, $S h\left(\mathbb{E}_{v}\right)+S h\left(\mathbb{E}_{\widehat{v}}\right)=\left(\frac{47}{72}, \frac{47}{72}, \frac{25}{36}\right) \neq S h\left(\mathbb{E}_{v+\widehat{v}}\right) . \diamond$

We extend the model in such a way that players have a belief about the likelihoods of the underlying fully defined games. First of all we need to formalize the concept of a belief in this context. Let $N$ be the set of players. The set $G^{N}$ of fully defined games with player set $N$ is isomorphic to $\mathbb{R}^{2^{N} \backslash\{\emptyset\}}$. Let $\mathcal{A}$ be the Lebesgue-Borel $\sigma$-algebra on $\mathbb{R}^{2^{N}} \backslash\{\emptyset\}$. This turns $G^{N}$ into a measurable space $\left(G^{N}, \mathcal{A}\right)$. The belief of the players can be expressed as a probability measure 
$P$ over $\left(G^{N}, \mathcal{A}\right)$. It is reasonable to restrict attention to those beliefs, of which the support

$$
\operatorname{Supp}(P):=\left\{w \in G^{N} \mid P(A)>0 \text { for each open } A \ni w\right\}
$$

contains only extensions of the game $v$. Furthermore, we assume that any coalition is expected to have a finite worth. For any $\mathcal{M}$-game $v$ let $\Pi^{v}$ be the set of all such probability measures $P$ over $\left(G^{N}, \mathcal{A}\right)$, i.e. that satisfy

$$
\operatorname{Supp}(P) \subseteq\left\{w \in G^{N}|w|_{\mathcal{M}}=v\right\}
$$

and

$$
-\infty<\int_{G^{N}} w(S) d P(w)<\infty \text { for all } S \subseteq N .
$$

We are now ready to define the set of all partially defined cooperative games with beliefs and player set $N$ by

$$
P G B^{N}:=\left\{(v, P) \mid v \in P G^{N} \text { and } P \in \Pi^{v}\right\} .
$$

Let $v$ be a fully defined game and

$$
\delta_{v}(A)=\left\{\begin{array}{cc}
1 & \text { if } v \in A \\
0 & \text { otherwise }
\end{array}, \text { for all } A \in \mathcal{A}\right.
$$

be the probability measure assigning probability 1 to the game $v$ itself. Such measures are called Dirac measures. Note that $\Pi^{v}=\left\{\delta_{v}\right\}$ for any fully defined game. This embeds $G^{N}$ into $P G B^{N}$ by identifying $w$ with $\left(w, \delta_{w}\right)$ for any fully defined cooperative game on $N$. Let

$$
P G B:=\bigcup\left\{P G B^{N}|\emptyset \neq N \subseteq \mathbb{N},| N \mid<\infty\right\}
$$

be the set of all partially defined cooperative games with beliefs. A value solution $f$ for partially defined cooperative games with beliefs is a function

$$
f: P G B^{N} \rightarrow \mathbb{R}^{N}
$$

\section{Extending the Shapley value: The expected Shapley value}

Next we present an extension of the Shapley value for fully defined games to partially defined games with beliefs. Young (1985) characterizes the Shapley 
value for fully defined games with efficiency, anonymity and strong monotonicity. We adapt these axioms to partially defined games with beliefs, and show that these adapted axioms give rise to a unique extension of the Shapley value to these games. As pointed out in example 1, linearity, especially additivity, may be troublesome to use in a characterization of such an extension of the Shapley value. An adaptation of linearity is discussed lateron in section 4 .

Let $f: P G B^{N} \rightarrow \mathbb{R}^{N}$ be a value solution. We call $f$ expectationally efficient if it does not distribute more than what the grand coalition $N$ will get in expectation, i.e.

$$
\sum_{i \in N} f_{i}\left(v, P^{v}\right)=\int_{G^{N}} w(N) d P^{v}(w)
$$

for any game $\left(v, P^{v}\right)$ in $P G B^{N}$. If the worth of the grand coalition is not known, then ex-post, i.e. when a fully defined game $\widehat{w}$ has been drawn from the carrier of $P^{v}$, ex-post efficiency

$$
\sum_{i \in N} f_{i}\left(v, P^{v}\right)=\widehat{w}(N)
$$

can be violated. Players could insure against this with a risk-neutral insurer who also beliefs that the true game is drawn from $P^{v}$. It might also be that the game is played repeatedly and all realizations are independent. Then excesses and shortfalls of the worth of the grand coalition will cancel out in the long run. Then zero cost for insurance and equal interest rates to finance shortfalls and invest excesses have to be assumed.

If $N \in \mathcal{M}$ only extensions $w$ where $w(N)=v(N)$ are in the carrier of the measure $P^{v}$, and the integral on the right hand side equals $v(N)$. So in that case expectational efficiency reduces to the equation

$$
\sum_{i \in N} f_{i}\left(v, P^{v}\right)=v(N) .
$$

So we will have ex-post efficiency in that case.

It turns out that all we need for our characterization is efficiency for fully defined games, i.e.

$$
\sum_{i \in N} f_{i}\left(v, P^{v}\right)=v(N),
$$

for all $v \in P B G^{N}$ with coalition list $\mathcal{M}=2^{N}$. Hence, accepting efficiency for fully defined games along with the other axioms used, implies acceptance of expectational efficiency. 
Let $\tau: N \rightarrow N$ be a permutation of the player set $N$. For any fully defined game $w \in G^{N}$ the permuted game of $w, w^{\tau}$ is the fully defined game given by

$$
w^{\tau}(S):=w\left(\tau^{-1}(S)\right) \text { for all } S \subseteq N,
$$

where

$$
\tau^{-1}(S):=\left\{\tau^{-1}(i) \mid i \in S\right\}
$$

Let $A \in \mathcal{A}$, then the permuted set $A^{\tau}$ is given by

$$
A^{\tau}:=\left\{w^{\tau} \mid w \in A\right\} .
$$

For the permuted $\sigma$-algebra $\mathcal{A}^{\tau}:=\left\{A^{\tau} \mid A \in \mathcal{A}\right\}$ we obviously have $\mathcal{A}^{\tau}=\mathcal{A}$. So, if $P$ is a probability measure on $\left(G^{N}, \mathcal{A}\right)$ we can define the permuted probability measure $P^{\tau}$ on $\left(G^{N}, \mathcal{A}\right)$ by

$$
P^{\tau}(A):=P\left(A^{\tau^{-1}}\right) \text { for all } A \in \mathcal{A} .
$$

Furthermore, we can also permute partially defined cooperative games, but then the coalition list has to be permuted also. So for any coalition list $\mathcal{M}$, let $\mathcal{M}^{\tau}$ be the permuted coalition list given by

$$
\mathcal{M}^{\tau}:=\{\tau(S) \mid S \in \mathcal{M}\} .
$$

If $\mathcal{M}$ is a symmetric coalition list, then $\mathcal{M}^{\tau}=\mathcal{M}$, and the set of known coalition sizes $K$ will not change. Let $v \in P G^{N}$ be a partially defined cooperative game with coalition list $\mathcal{M}$, then the permuted game $v^{\tau}$ of $v$ is the $\mathcal{M}^{\tau}$-game given by

$$
v^{\tau}(S):=v\left(\tau^{-1}(S)\right) \text { for all } S \in \mathcal{M}^{\tau} .
$$

Finally, for any game $\left(v, P^{v}\right) \in P G B^{N}$, we call $\left(v^{\tau},\left(P^{v}\right)^{\tau}\right)$ the permuted game of $\left(v, P^{v}\right)$ under $\tau$. Obviously, we have $\left(v^{\tau},\left(P^{v}\right)^{\tau}\right) \in P G B^{N}$.

We call the value solution $f: P G B^{N} \rightarrow \mathbb{R}^{N}$ anonymous if

$$
f\left(\left(v^{\tau},\left(P^{v}\right)^{\tau}\right)\right)=f^{\tau}\left(v, P^{v}\right):=\left(f_{\tau^{-1}(i)}\left(v, P^{v}\right)\right)_{i \in N}
$$

for any permutation $\tau$ of the player set and all $\left(v, P^{v}\right) \in P G B^{N}$. We call $f$ anonymous for fully defined games if we only demand this property to hold for fully defined games. Note that $\left|\Pi^{v}\right|=\left|\left\{\delta_{v}\right\}\right|=1$ for all such $v \in G^{N}$, so $\left.f\right|_{G^{N}}$ does not depend on the belief. Hence, anonymity for fully defined games is just the usual anonymity condition for a value solution $g: G^{N} \rightarrow \mathbb{R}^{N}$, i.e. 
$g\left(w^{\tau}\right)=\left(g_{\tau^{-1}(i)}(w)\right)_{i \in N}$ for all $w \in G^{N}$ and all permutations $\tau$ of the player set $N$.

In order to define strong monotonicity for $\mathcal{M}$-games with beliefs we use the marginal contributions operator $\Delta_{i}(w): 2^{N} \backslash\{\emptyset\} \rightarrow \mathbb{R}$ of a player $i \in N$ for fully defined games $w$ given by

$$
\Delta_{i}(w)(S):=w(S \cup\{i\})-w(S), S \in 2^{N} \backslash\{\emptyset\} .
$$

For a game $\left(v, P^{v}\right) \in P G B^{N}$, and a player $i \in N$, let the (expected) marginal contributions operator $\Delta_{i}\left(v, P^{v}\right): 2^{N} \backslash\{\emptyset\} \rightarrow \mathbb{R}$ be given by

$$
\Delta_{i}\left(v, P^{v}\right)(S):=\int_{G^{N}} \Delta_{i}(w)(S) d P^{v}(w) .
$$

The expected marginal contributions operator extends $\Delta_{i}$ to partially defined cooperative games with beliefs. Using the (expected) marginal contributions operator for $\mathcal{M}$-games with beliefs, we say that the value solution $f$ is strongly monotonic if for all games $\left(v, P^{v}\right),\left(\widetilde{v}, P^{\widetilde{v}}\right) \in P G B^{N}$ we have that if

$$
\Delta_{i}\left(v, P^{v}\right) \geq \Delta_{i}\left(\widetilde{v}, P^{\widetilde{v}}\right),
$$

for an $i \in N$, then

$$
f_{i}\left(v, P^{v}\right) \geq f_{i}\left(\widetilde{v}, P^{\widetilde{v}}\right) .
$$

The expected Shapley value $S h\left(v, P^{v}\right)$ of a partially defined game $\left(v, P^{v}\right) \in$ $P G B^{N}$ is the value solution given by

$$
S h\left(v, P^{v}\right):=\int_{G^{N}} \operatorname{Sh}(w) d P^{v}(w) .
$$

Given any $\mathcal{M}$-game $v$ with belief $P^{v}$ we can define the expected game $\mathbb{E}_{v}$ of $v$ under that belief by

$$
\mathbb{E}_{v}(S):=\left\{\begin{array}{cc}
v(S) & \text { for all } S \in \mathcal{M}, \\
\int_{G^{N}} w(S) d P^{v}(w) & \text { otherwise. }
\end{array}\right.
$$

Then the expected Shapley value of $\left(v, P^{v}\right)$ equals the Shapley value of the fully defined game $\mathbb{E}_{v}$. This follows by the linearity of the integral and the linearity of the Shapley value for fully defined games. Furthermore, linearity of the integral and of the marginal contributions operator for fully defined games imply that $\Delta_{i}\left(v, P^{v}\right)(S)=\Delta_{i}\left(\mathbb{E}_{v}\right)(S)$. We have $P^{v}=\delta_{v}$ for all $\left(v, P^{v}\right) \in G^{N}$ and so for fully defined games we obtain $v=\mathbb{E}_{v}$. Thus the expected Shapley value 
equals the Shapley value for fully defined games. Note that we write $S h\left(v, P^{v}\right)$, $v \in P G B^{N}$ for the expected Shapley value, and $S h(w), w \in G^{N}$ for the Shapley value.

Theorem 2 Let $f: P G B^{N} \rightarrow \mathbb{R}^{N}$ be a value solution that is efficient for fully defined games, anonymous for fully defined games, and strongly monotonic. Then $f$ is the expected Shapley value.

Proof. We use the characterization of Young (1985), who showed that any value solution for fully defined games satisfying efficiency, anonymity and strong monotonicity is the Shapley value on $G^{N}$. So

$$
\left.f\right|_{G^{N}}=\left.S h\right|_{G^{N}} .
$$

Let $\mathbb{E}_{v}$ be the expected game of $\left(v, P^{v}\right),\left(v, P^{v}\right) \in P B G^{N}$. This defines a fully defined game. Let $i \in N$. As

$$
\begin{aligned}
\Delta_{i}\left(v, P^{v}\right)(S) & =\int_{G^{N}} \Delta_{i}(w)(S) d P^{v}(w) \\
& =\int_{G^{N}}(w(S \cup\{i\})-w(S)) d P^{v}(w) \\
& =\int_{G^{N}} w(S \cup\{i\}) d P^{v}(w)-\int_{G^{N}} w(S) d P^{v}(w) \\
& =\Delta_{i}\left(\mathbb{E}_{v}, \delta_{\mathbb{E}_{v}}\right)(S),
\end{aligned}
$$

we obtain by strong monotonicity and equation $(*)$, that

$$
\begin{aligned}
f_{i}\left(v, P^{v}\right) & =f_{i}\left(\mathbb{E}_{v}, \delta_{\mathbb{E}_{v}}\right) \\
& =S h_{i}\left(\mathbb{E}_{v}\right) .
\end{aligned}
$$

As noted when defining the expected Shapley value we have by the linearity of the Shapley value and the linearity of the integral that

$$
S h_{i}\left(\mathbb{E}_{v}\right)=f_{i}\left(v, P^{v}\right) .
$$

The expected Shapley value is not only efficient for fully defined games, but also expectationally efficient and anonymous for any partially defined cooperative game.

Lemma 3 The expected Shapley value is expectationally efficient and anonymous. 
Proof. Let $\left(v, P^{v}\right) \in P G B^{N}$. We have $S h_{i}\left(v, P^{v}\right)=S h_{i}\left(\mathbb{E}_{v}\right)$ for all $i \in N$, and by expectational efficiency for fully defined games we obtain

$$
\sum_{i \in N} S h_{i}\left(v, P^{v}\right)=\sum_{i \in N} S h_{i}\left(\mathbb{E}_{v}\right)=\mathbb{E}_{v}(N)=\int_{G^{N}} w(N) d P^{v}(w) .
$$

For anonymity, note that by anonymity for fully defined games, the substitution rule and $d\left(P^{v}\right)^{\tau}(w)=d P^{v}\left(w^{\tau^{-1}}\right)$, we have that

$$
\begin{aligned}
\operatorname{Sh}\left(v^{\tau},\left(P^{v}\right)^{\tau}\right) & =\int_{G^{N}} \operatorname{Sh}(w) d\left(P^{v}\right)^{\tau}(w) \\
& =\int_{G^{N}} \operatorname{Sh}(w) d P^{v}\left(w^{\tau^{-1}}\right) \\
& =\int_{\left(G^{N}\right)^{--1}} \operatorname{Sh}\left(w^{\tau}\right) d P^{v}(w) \\
& =\int_{G^{N}} S h^{\tau}(w) d P^{v}(w) \\
& =S h^{\tau}\left(v, P^{v}\right) .
\end{aligned}
$$

\section{Linearity of the expected Shapley value}

In this section we shall define a weaker form of linearity, and give a characterization of the expected Shapley value with that weaker form of linearity, dummy player, efficiency and anonymity. First we note that partially defined games with the same coalition list can be added coalitionwise. The value solution $f: P G B^{N} \rightarrow \mathbb{R}^{N}$ is said to be strongly linear if for all $\alpha, \beta \in \mathbb{R}$ and $\mathcal{M}$-games $v, \widehat{v}$ we have that

$$
f(\alpha v+\beta \widehat{v})=\alpha f(v)+\beta f(\widehat{v}) .
$$

Example 1 showed that the expected Shapley value does not satisfy strong linearity. However, taking beliefs into account may result in a linearity condition which is satisfied by the expected Shapley value. Moreover, we can define an addition operator on $P G B^{N}$ also for games with different coalition lists.

For any $\alpha \in \mathbb{R}$ and any partially defined game $\left(v, P^{v}\right)$ let $\bar{P}^{\alpha v}$ be the probability measure defined for $A \in \mathcal{A}$ by $\bar{P}^{\alpha v}(A)=P^{v}\left(\frac{1}{\alpha} A\right)$ if $\alpha \neq 0$, and $\bar{P}^{\alpha v}=\delta_{\underline{0}}$, if $\alpha=0$, where $\underline{0}$ is the fully defined zero-game, in which all coalitions receive 0 . For any two partially defined games $\left(v, P^{v}\right),\left(\widehat{v}, P^{\widehat{v}}\right)$, let $\bar{P}^{v+\widehat{v}}$ be the probability 
measure obtained by convoluting $P^{v}$ and $P^{\widehat{v}}$, i.e. for every $A \in \mathcal{A}$

$$
\bar{P}^{v+\widehat{v}}(A)=\int_{G^{N}} \int_{G^{N}} \chi_{A}(x+y) d P^{v}(x) d P^{\widehat{v}}(y),
$$

where

$$
\chi_{A}(x+y)=\left\{\begin{array}{cc}
1 & \text { if } x+y \in A \\
0 & \text { otherwise }
\end{array}\right.
$$

In words, $\bar{P}^{v+\widehat{v}}(A)$ is the probability that the sum of two extensions of the games $\left(v, P^{v}\right)$ and $\left(\widehat{v}, P^{\widehat{v}}\right)$ respectively is in $A$, given that the extensions are independently distributed according to $P^{v}$ and $P^{\widehat{v}}$ respectively.

Let $\left(v, P^{v}\right),\left(\widehat{v}, P^{\widehat{v}}\right) \in P G B^{N}$ and $\alpha \in \mathbb{R}$. The games $v$ and $\widehat{v}$ can be added coalitionwise where the worths of both games are known, i.e. on the coalition list $\mathcal{M}:=\operatorname{dom}(v) \cap \operatorname{dom}(\widehat{v})$. We define an addition in $P G B^{N}$ by

$$
\left(v, P^{v}\right) \oplus\left(\widehat{v}, P^{\widehat{v}}\right):=\left(\left(\left.v\right|_{\mathcal{M}}+\left.\widehat{v}\right|_{\mathcal{M}}\right), \bar{P}^{v+\widehat{v}}\right) .
$$

The scalar multiplication in $P G B^{N}$ is defined by

$$
\alpha \circledast\left(v, P^{v}\right):=\left(\alpha v, \bar{P}^{\alpha v}\right) .
$$

We call a value solution $f$ weakly linear if

$$
f\left(\alpha \circledast\left(v, P^{v}\right) \oplus \beta \circledast\left(\widehat{v}, P^{\widehat{v}}\right)\right)=\alpha f\left(v, P^{v}\right)+\beta f\left(\widehat{v}, P^{\widehat{v}}\right),
$$

for all $\alpha, \beta \in \mathbb{R}$ and $\left(v, P^{v}\right),\left(\widehat{v}, P^{\widehat{v}}\right) \in P G B^{N}$. So the additional constraint weakening linearity is on the belief for the game $\left.\alpha v\right|_{\mathcal{M}}+\left.\beta \widehat{v}\right|_{\mathcal{M}}$.

Next it is shown that the expected Shapley value satisfies weak linearity.

Theorem 4 The expected Shapley value is weakly linear. 
Proof. Let $\left(v, P^{v}\right),\left(w, P^{w}\right) \in P G B^{N}$. The expected Shapley value satisfies

$$
\begin{aligned}
\operatorname{Sh}\left(\left(v, P^{v}\right) \oplus\left(w, P^{w}\right)\right) & =\int_{G^{N}} \operatorname{Sh}(x) d \bar{P}^{v+w}(x) \\
& =\int_{G^{N}} \int_{G^{N}} \operatorname{Sh}(x-y+y) d P^{v}(x-y) d P^{w}(y) \\
& =\int_{G^{N}} \int_{G^{N}}(\operatorname{Sh}(x-y)+S h(y)) d P^{v}(x-y) d P^{w}(y) \\
& =\int_{G^{N}}\left[\left(\int_{G^{N}-t} \operatorname{Sh}(\widetilde{x}) d P^{v}(\widetilde{x})\right)+S h(y)\right] d P^{w}(y) \\
& =\int_{G^{N}}\left[\left(\int_{G^{N}} \operatorname{Sh}(\widetilde{x}) d P^{v}(\widetilde{x})\right)+S h(y)\right] d P^{w}(y) \\
& =\int_{G^{N}}\left[S h\left(v, P^{v}\right)+S h(y)\right] d P^{w}(y) \\
& =S h\left(v, P^{v}\right) P^{w}\left(G^{N}\right)+\int_{G^{N}} \operatorname{Sh}(y) d P^{w}(y) \\
& =S h\left(v, P^{v}\right)+S h\left(w, P^{w}\right) .
\end{aligned}
$$

Let $\alpha \in \mathbb{R}$. We are done if we can show that the expected Shapley value also satisfies $S h\left(\alpha \circledast\left(v, P^{v}\right)\right)=\alpha S h\left(v, P^{v}\right)$. This follows as for $\alpha \neq 0$ we have

$$
\begin{aligned}
S h\left(\alpha \circledast\left(v, P^{v}\right)\right) & =\int_{G^{N}} x d \bar{P}^{\alpha v}(x) \\
& =\int_{G^{N}} x d P^{v}\left(\frac{1}{\alpha} x\right) \\
& =\int_{\frac{1}{\alpha} G^{N}} \alpha x d P^{v}(x) \\
& =\alpha \int_{G^{N}} x d P^{v}(x) \\
& =\alpha \operatorname{Sh}\left(v, P^{v}\right),
\end{aligned}
$$

and for $\alpha=0$ we have

$$
S h\left(\alpha \circledast\left(v, P^{v}\right)\right)=S h\left(\underline{0}, \delta_{0}\right)=(0, \ldots, 0)=0 S h\left(v, P^{v}\right) .
$$

The expected Shapley value can also be characterized with weak linearity. One of the standard axioms that we will need is the null player axiom. Let $\left(v, P^{v}\right) \in P G B^{N}$. We call a player $i \in N$ a null player in $\left(v, P^{v}\right)$ if the (expected) marginal contributions of that player are 0 , i.e. if

$$
\Delta_{i}\left(v, P^{v}\right)(S)=0 \text { for all } S \subseteq N .
$$


We say that the value solution $f: P G B^{N} \rightarrow \mathbb{R}^{N}$ satisfies the null player property, if for all $\left(v, P^{v}\right) \in P G B^{N}$, and all null players $i$ in the game $\left(v, P^{v}\right)$, we have

$$
f_{i}\left(v, P^{v}\right)=0 .
$$

Theorem 5 Let $f: P G B^{N} \rightarrow \mathbb{R}^{N}$ be a value solution satisfying efficiency, anonymity, null player and weak linearity. Then $f$ is the expected Shapley value.

Proof. For fully defined games weak linearity and strong linearity are the same. Shapley (1953) has characterized the Shapley value for fully defined games with efficiency, anonymity, null player and strong linearity. So

$$
\left.f\right|_{G^{N}}=\left.S h\right|_{G^{N}} .
$$

Let $\left(v, P^{v}\right)$ be a partially defined cooperative game with coalition list $\mathcal{M}$. Let $\delta_{\mathbb{E}_{v}}$ be the Dirac measure on the expected game $\mathbb{E}_{v}$ of $\left(v, P^{v}\right)$. The expected game $\mathbb{E}_{z}$ of the game

$$
\left(z, P^{z}\right):=\left(v, P^{v}\right) \oplus\left((-1) \otimes\left(\mathbb{E}_{v}, \delta_{\mathbb{E}_{v}}\right)\right)
$$

satisfies

$$
\begin{aligned}
\mathbb{E}_{z}(S) & =\int_{G^{N}} \int_{G^{N}}(x+y) d P^{v}(x) d\left(\delta_{\mathbb{E}_{v}}\right)(-y) \\
& =\int_{G^{N}} \int_{G^{N}}(x-\widetilde{y}) d P^{v}(x) d\left(\delta_{\mathbb{E}_{v}}\right)(\widetilde{y}) \\
& =\int_{G^{N}}\left(\mathbb{E}_{v}-y\right) d\left(\delta_{\mathbb{E}_{v}}\right)(y) \\
& =0
\end{aligned}
$$

for all $S \in 2^{N}$. As $\Delta_{i}\left(v, P^{v}\right)=\Delta_{i}\left(\mathbb{E}_{z}\right)$ this shows that all players in $\left(z, P^{z}\right)$ are null players. Hence,

$$
f\left(z, P^{z}\right)=(0, \ldots, 0) .
$$

As $\left(z, P^{z}\right)$ was defined to be

$$
\left(\left(v, P^{v}\right) \oplus(-1) \circledast\left(\mathbb{E}_{v}, \delta_{\mathbb{E}_{v}}\right)\right)
$$

this implies by weak linearity that

$$
f\left(v, P^{v}\right)-f\left(\mathbb{E}_{v}, \delta_{\mathbb{E}_{v}}\right)=(0, \ldots, 0) .
$$


As $\left(\mathbb{E}_{v}, \delta_{\mathbb{E}_{v}}\right)$ can be identified with the fully defined game $\mathbb{E}_{v} \in G^{N}$ we have proved using $(*)$ that

$$
f\left(v, P^{v}\right)=S h\left(\mathbb{E}_{v}, \delta_{\mathbb{E}_{v}}\right) .
$$

Again, $S h\left(\mathbb{E}_{v}, \delta_{\mathbb{E}_{v}}\right)$ equals the expected Shapley value $S h\left(v, P^{v}\right)$.

The proof uses efficiency and anonymity only on fully defined games. Weak linearity can be weakened to weak additivity, just as in the corresponding theorem of Shapley (1953).

\section{Representation, Dividends and Potential}

Some important results for fully defined games concern representation by unanimity games, dividend and potential. This section will show how to extend these results to partially defined cooperative games with beliefs.

First we look at the representation by unanimity games. For nonempty subsets $T$ of $N$ the T-unanimity game is a fully defined game, say $u_{T}$, defined by

$$
u_{T}(S)=\left\{\begin{array}{cc}
1 & \text { if } T \subseteq S \\
0 & \text { otherwise }
\end{array}\right.
$$

For any fully defined game $w$ we have the representation

$$
w=\sum_{T \in 2^{N} \backslash\{\emptyset\}} c_{T} u_{T}
$$

where

$$
c_{T}=\sum_{S: S \subseteq T}(-1)^{|T|-|S|} w(S) .
$$

The set $\left\{u_{T} \mid T \in 2^{N} \backslash\{\emptyset\}\right\}$ is linearly independent, so it forms a basis for the set of fully defined games. The question arises whether a representation with unanimity games is also possible for partially defined games with beliefs.

Let $C: G^{N} \rightarrow \mathbb{R}^{2^{N} \backslash\{\emptyset\}}$ be the mapping given by $C(w)=\left(c_{T}\right)_{T \in 2^{N} \backslash\{\emptyset\}}$, whenever $w=\sum_{T \in 2^{N} \backslash\{\emptyset\}} c_{T} u_{T}$. Then $C$ is bijective and measurable as a mapping from $\left(G^{N}, \mathcal{A}\right)$ to $\left(\mathbb{R}^{2^{N} \backslash\{\emptyset\}}, \mathcal{A}\right)$. Let $\left(v, P^{v}\right)$ be a partially defined cooperative game with beliefs. The probability measure $P^{C}$ on $\left(\mathbb{R}^{2^{N} \backslash\{\emptyset\}}, \mathcal{A}\right)$ given by $P^{C}:=P^{v} \circ C^{-1}$, represents $P^{v}$ in the following sense:

$$
\begin{aligned}
P^{v}(A) & =\left(P^{v} \circ C^{-1} \circ C\right)(A) \\
& =P^{C}(C(A)) \text { for all } A \in \mathcal{A} .
\end{aligned}
$$


So, beliefs about the underlying fully defined game can also be seen as beliefs about possible representations of these underlying games by unanimity games.

Next we want to extend the concept of a dividend to partially defined cooperative games with beliefs. Let $w$ be a fully defined game. For each coalition $T$, Harsanyi (1959) defined a real number $d_{T}$, the dividend, in a recursive manner as follows.

$$
d_{T}(w):=\left\{\begin{array}{cc}
0 & \text { if } T=\emptyset, \\
|T|^{-1}\left(w(T)-\sum_{S \subsetneq T}|S| d_{S}(w)\right) & \text { if }|T| \geq 1 .
\end{array}\right.
$$

If $w=\sum c_{T} u_{T}$ then $|T| d_{T}(w)=c_{T}$ for all $T \neq \emptyset$ and $S h_{i}(w)=\sum_{T: i \in T} d_{T}(w)$, i.e. the Shapley value of a player is the sum of all dividends of coalitions to which the player belongs.

Taking into account the beliefs of the players about the underlying games, will turn dividends into random variables, with a probability measure depending on the beliefs of the players. Let $\left(v, P^{v}\right)$ be a partially defined cooperative game. Let $C: G^{N} \rightarrow \mathbb{R}^{2^{N} \backslash\{\emptyset\}}$ and $P^{C}$ be as defined above. Let $D: G^{N} \rightarrow \mathbb{R}^{2^{N} \backslash\{\emptyset\}}$ be the mapping given by $D(w)=\left(d_{T}(w)\right)_{T}$. Then $D$ is bijective and measurable as a mapping from $\left(G^{N}, \mathcal{A}\right)$ to $\left(\mathbb{R}^{2^{N}} \backslash\{\emptyset\}, \mathcal{A}\right)$. Defining $P^{D}$ as the probability measure on $\left(\mathbb{R}^{2^{N} \backslash\{\emptyset\}}, \mathcal{A}\right)$ such that $P^{D}:=P^{v} \circ D^{-1}$ gives the desired analogy for the random dividend $D:\left(G^{N}, \mathcal{A}\right) \rightarrow\left(\mathbb{R}^{2^{N} \backslash\{\emptyset\}}, \mathcal{A}\right)$ distributed according to $P^{D}$. As the reader can verify using the corresponding facts for fully defined games we have

1. the mapping $F: \mathbb{R}^{2^{N} \backslash\{\emptyset\}} \rightarrow \mathbb{R}^{2^{N} \backslash\{\emptyset\}}$ given by $F(d):=\left(|T| d_{T}\right)_{T \in \mathbb{R}^{2} \backslash\{\emptyset\}}$ is bijective and measurable as a mapping from $\left(\mathbb{R}^{2^{N} \backslash\{\emptyset\}}, \mathcal{A}\right)$ to $\left(\mathbb{R}^{2^{N} \backslash\{\emptyset\}}, \mathcal{A}\right)$.

2. $F(D)=C$, i.e. $P^{D}(A)=P^{C}(F(A))$ for any $A \in \mathcal{A}$.

3.

$$
\begin{aligned}
S h_{i}\left(v, P^{v}\right) & =\sum_{T: i \in T} \int_{G^{N}}(D(w))_{T} d P^{v}(w) \\
& =\sum_{T: i \in T} \int_{\mathbb{R}} d_{T} d P^{D_{T}}\left(d_{T}\right)
\end{aligned}
$$

where the measure $P^{D_{T}}$ on $\mathbb{R}$ is the image of the measure $P^{D}$ under the projection on the coordinate (coalition) $T$, i.e.

$$
P^{D_{T}}(B)=P^{D}\left(\left\{x \in \mathbb{R}^{2^{N}} \mid x_{T} \in B\right\}\right)
$$


for all Lebesgue-Borel measurable sets $B \subseteq \mathbb{R}$.

The last extension concerns the potential of a game. A potential (Hart and Mas-Colell, 1989) is a real valued function $Q: G \rightarrow \mathbb{R}$ such that

$$
\begin{aligned}
Q(\emptyset, w) & =0 \\
\sum_{i \in N} D_{i} Q(N, w) & =w(N) \text { for all } w \in G^{N} .(Q \text {-efficiency })
\end{aligned}
$$

Here $D_{i} Q(N, w)=Q(N, w)-Q\left(N \backslash\{i\},\left.w\right|_{N \backslash\{i\}}\right)$. If $Q$ is a potential then the gradient $\operatorname{grad} Q(N, w):=\left(D_{i} Q(N, w)\right)_{i \in N}$ is an efficient payoff vector for the game. By Q-efficiency

$$
Q(N, w)=\frac{1}{|N|}\left(w(N)+\sum_{i \in N} Q\left(N \backslash\{i\},\left.w\right|_{N \backslash\{i\}}\right)\right) .
$$

So the potential of $w \in G^{N}$ can be determined by the potentials of the subgames. By $Q(\emptyset, w)=0$ it is therefore uniquely defined. The potential is connected to the dividends and the Shapley value of fully defined games in the following way (Hart and Mas-Colell, 1989):

$$
\begin{aligned}
Q(N, w) & =\sum_{\emptyset \neq T \subseteq N} d_{T} \\
\operatorname{grad} Q(N, w) & =S h(w) .
\end{aligned}
$$

Let $\left(v, P^{v}\right)$ be a partially defined cooperative game with player set $N$ and coalition list $\mathcal{M}$. The restriction of $w$ to the player set $\widetilde{N} \subseteq N$ is the partially defined cooperative game $\left(v \mid \widetilde{N}, P^{v \mid \widetilde{N}}\right)$ with player set $\widetilde{N}$, coalition list $\widetilde{\mathcal{M}}=\mathcal{M} \cap 2^{\widetilde{N}}$ and belief given by

$$
P^{\left.v\right|_{\widetilde{N}}}(A)=P^{v}\left(\left\{x \in \mathbb{R}^{2^{N} \backslash\{\emptyset\}}|x|_{\widetilde{\mathcal{M}}} \in A\right\}\right)
$$

for all $A$ in the Lebesgue-Borel $\sigma$-algebra on $\mathbb{R}^{2^{\widetilde{N}} \backslash\{\emptyset\}}$. A potential on the set of partially defined cooperative games with beliefs is a real valued function $Q: P G B \rightarrow \mathbb{R}$. The result for fully defined games can be extended to hold also for partially defined games when requiring instead of $Q$-efficieny

$$
\sum_{i \in N} D_{i} Q\left(N, v, P^{v}\right)=\int_{G^{N}} w(N) d P^{v}(w)(\text { expected } Q \text {-efficieny) }
$$

for all partially defined games $\left(v, P^{v}\right)$ with player set $N$. Define $D_{i} Q\left(N, v, P^{v}\right)$, $\operatorname{grad} Q\left(N, v, P^{v}\right)$ just as for fully defined games. The gradient gives us by expectational $Q$-efficiency an expectationally efficient payoff vector. Furthermore, 
the potential is uniquely defined, extends the potential for fully defined games and can be connected to the expected Shapley value and expectations of the random dividends for partially defined games in a similar manner as for fully defined games.

\section{Reduced and normalized Shapley Value}

This section shows how to embed the reduced Shapley value (Willson, 1993) and the normalized Shapley value (Housman, 2001) into our model. Both these values are only defined for symmetric coalition lists containing the grand coalition, and do not depend on the belief. So we can restrict attention to

$$
\widehat{P G}^{N}:=\left\{v \in P G^{N} \mid \operatorname{dom}(v) \text { is a symmetric coalition list, } N \in \operatorname{dom}(v)\right\} .
$$

Let $n:=|N|$ be the cardinality of the player set $N$. The reduced Shapley value of a game $v$ in $\widehat{P G}^{N}$ is given by

$$
R S h_{i}(v):=\frac{1}{n} \sum_{m \in K}\left(\left(\begin{array}{c}
n-1 \\
m-1
\end{array}\right)^{-1} \sum_{\substack{|S|=m \\
i \in S}} v(S)-\left(\begin{array}{c}
n-1 \\
m
\end{array}\right)^{-1} \sum_{\substack{|S|=m \\
i \notin S}} v(S)\right)
$$

for any player $i \in N$, where $K$ are the known coalition sizes. This can also be written in terms of marginal (known) contributions. Let

$$
\rho(m):=\max \{k \in K \mid k<m\}
$$

be the largest known coalition size smaller than $m \in K$. The marginal known contributions of a player $i \in N$ to a coalition $S \ni i,|S| \in K$, are then given by $w(S)-w(R)$ for any $R \subseteq S-\{i\},|R|=\rho(|S|)$. Willson (1993) characterizes the reduced Shapley value by a strong monotonicity condition based on these marginal contributions, together with efficiency, strong anonymity and strong linearity. Housman (2001) notes that it can also be expressed using these marginal contributions as

$$
R S h_{i}(v)=\frac{1}{n} \sum_{m \in K}\left(\begin{array}{c}
n-1 \\
m-1
\end{array}\right)^{-1} \sum_{\substack{|S|=m \\
i \in S}}\left(\begin{array}{c}
m-1 \\
\rho(m)
\end{array}\right) \sum_{\substack{|R|=\rho(m) \\
R \subseteq S-\{i\}}}(w(S)-w(R))
$$

for any player $i \in N$. Willson (1993) and Housman (2001) also note that the reduced Shapley value of $v \in \widehat{P G}^{N}$ equals the Shapley value of the extension $w$, 
where the unknown coalition worths are set equal to a constant which is allowed to depend on the size, i.e. $\left.w\right|_{\mathcal{M}}=v$ and $w(S):=c_{|S|}$ for all $S \notin \mathcal{M}$, where $c_{k} \in \mathbb{R}$ for all $k \in\{0, \ldots, n\} \backslash K$. The Shapley value will then be independent of the chosen constants, the corresponding terms cancel out and only the terms forming the reduced Shapley value influence the value. Using that, we can express the reduced Shapley value as an expected Shapley value.

Lemma 6 Let $v \in \widehat{P G}^{N}$. Let $P^{v} \in \Pi^{v}$ be a belief leading to an expected game $\mathbb{E}_{v}$ satisfying

$$
\mathbb{E}_{v}(S)=\mathbb{E}_{v}(T)
$$

for all $S, T \subseteq N$ such that $|S|=|T| \in\{0, \ldots, n\} \backslash K$. Then $S h\left(v, P^{v}\right)=R S h(v)$.

Proof. This is just another way of saying that the unknown coalition worths are equal to a size-dependent constant.

It is not hard to find beliefs satisfying this lemma. Just take the Dirac measure on an extension where coalitions of the same size have the same value if their worths are unknown. The lemma says that the reduced Shapley value amounts to assuming implicitly that players believe that extensions, which are constant across unknown worths of coalitions of the same size, give good estimates of the missing values. That this can be problematic was already known to Willson (1993), who notes that usually such extensions will not satisfy properties such as monotonicity or superadditivity. Housman (2001) gives an example where the reduced Shapley value of a game is not among the Shapley values of any of its superadditive extensions. So, if players have such beliefs about the underlying game it might not be reasonable to use the reduced Shapley value.

Housman (2001) defined the normalized Shapley value for player $i \in N$ by

$$
\begin{aligned}
N S h_{i}(v)= & \frac{1}{n} \sum_{m \in K}\left(\left(\begin{array}{c}
n-1 \\
m-1
\end{array}\right)^{-1} \sum_{\substack{|S|=m \\
i \in S}} v(S)-\left(\begin{array}{c}
n-1 \\
m
\end{array}\right)^{-1} \sum_{\substack{|S|=m \\
i \notin S}} v(S)\right) \\
& +\left(1-\frac{|K|}{n}\right)\left(v(i)-\frac{1}{n-1} \sum_{j \in N \backslash\{i\}} v(j)\right),
\end{aligned}
$$

as an alternative if $1 \in K$. If $1 \notin K$ then the normalized Shapley value is defined to equal the reduced Shapley value, i.e. the last line of the sum disappears. The following lemma shows that the normalized Shapley value can also be obtained 
as an expected Shapley value, and to which estimates of the missing worths this amounts. It is stated only for symmetric coalition lists where $1 \in K$ (individual worths are known), as the normalized Shapley value equals the reduced Shapley value for games where $1 \notin K$.

Lemma 7 Let $v \in \widehat{P G}^{N}$ and suppose that $1 \in K$. Let $P^{v} \in \Pi^{v}$ be a belief leading to an expected game $\mathbb{E}_{v}$ satisfying

$$
\mathbb{E}_{v}(S)=\sum_{i \in S} v(\{i\})
$$

for all $S$ such that $S \notin \mathcal{M}$. Then $\operatorname{Sh}\left(v, P^{v}\right)=N \operatorname{Sh}(v)$.

Proof. Straightforward but tedious.

Using Dirac measures as beliefs it is not hard to specify beliefs satisfying the lemma. But again the expected games will usually violate common assumptions players might have. However, in a sense the lemma reveals that these estimates are a bit more sophisticated. They take into account at least the individual worths of the players, will in general not be constant across coalitions of the same size, and grow with coalition size if individual worths are positive. This method is appropiate for example if players believe they are playing additive games, and could probably still be used for beliefs, which are 'not too far away' from the belief to play an additive game. However, there is no intuitive reason why these worths should be used in every case. One could imagine many other, even more sophisticated estimates, e.g.

$$
\begin{aligned}
& w(S)=\max \left\{\sum_{T \in \mathcal{P}} v(T) \mid \mathcal{P} \text { any partition of } S \text { into sets } T, T \in \mathcal{M}\right\}, \\
& w(S)=\min \left\{\sum_{T \in \mathcal{P}} v(T) \mid \mathcal{P} \text { any partition of } S \text { into sets } T, T \in \mathcal{M}\right\},
\end{aligned}
$$

or $w(S)$ being any weighted average of the sum of coalition worths in such partitions. All of these can be accomodated as expected Shapley values with respect to specific beliefs. Whether there are axioms characterizing the corresponding Shapley values on $\widehat{P G}^{N}$ in the spirit of Willson (1993) and Housman (2001), i.e. without beliefs, is an open problem.

As mentioned already, Housman (2001) gave an example where the reduced Shapley value does not equal the Shapley value of any of the superadditive extensions of the partially defined cooperative game under consideration. It is 
not hard to imagine that the same problem arises with the normalized Shapley value. There the extension which is implicitly assumed to be the expected game might not be superadditive. The expected Shapley value behaves better in that sense, i.e. it picks a value from the set of values of the extensions, if one imposes a restriction on the support of the beliefs.

Lemma 8 Let $\left(v, P^{v}\right)$ be a partially defined cooperative game. Suppose that the support of $P^{v}$ is convex. Then the expected Shapley value of $\left(v, P^{v}\right)$ is equal to the Shapley value of an extension $w \in G^{N}$ of $v$, considered possible by the players, i.e.

$$
\operatorname{Sh}\left(v, P^{v}\right) \in\left\{\operatorname{Sh}(w) \mid w \in \operatorname{Supp}\left(P^{v}\right)\right\} .
$$

Proof. As the support $\operatorname{Supp}\left(P^{v}\right)$ of $P^{v}$ is convex we have $\mathbb{E}_{v} \in \operatorname{Supp}\left(P^{v}\right)$. Hence, $S h\left(v, P^{v}\right)=S h\left(\mathbb{E}_{v}\right) \in\left\{S h(w) \mid w \in \operatorname{Supp}\left(P^{v}\right)\right\}$.

This restriction is not unnatural and satisfied in important situations, such as monotonic, superadditive and convex extensions, or any other set of extensions arising from $l$ homogeneous linear inequalities,

$$
H w \geq 0, H \in \mathbb{R}^{l \times 2^{N} \backslash\{\emptyset\}}, l \text { any nonnegative integer, }
$$

on the vector $(w(S))_{S \in 2^{N} \backslash\{\emptyset\}}$ of coalition worths.

\section{Conclusions}

Adding belief to Willson's (1993) and Housman's (2001) definitions on partially defined cooperative games was shown to evolve into the existence of a natural and unique extension of the Shapley value to such games. It is defined for any coalition list and any belief having finite expectations of coalitional worths. It can be characterized by properties analogous to these of Young (1985) or Shapley (1953). Representation with unanimity games, dividend and potential can be extended to partially defined cooperative games with beliefs. The reduced Shapley value and the normalized Shapley can be incorporated as special cases. Housman (2001) already concluded, future research might consider situations where players have the option to choose among several coalition lists. This might be the case in practise, if players can, due to cost and complexity, only determine a limited number of worths. In that case it is of interest to investigate which worths should be determined. Another topic could be to extend the core to partially defined cooperative games. 


\section{References}

[1] Harsanyi JC (1959) A Bargaining Model for the Cooperative n-person Game. In Tucker AW and Luce RD (eds.) Contributions to the Theory of Games IV, Annals of Mathematics Studies No. 40, Princeton University Press, Princeton, New Jersey, pp. 324-356

[2] Hart S, and Mas-Colell A (1989) Potential, Value, and Consistency. Econometrica 57:589-614

[3] Housman D (2001) Linear and symmetric allocation methods for partially defined cooperative games. Int J Game Theory 30:377-404

[4] Shapley LS (1953) A Value for n-person games. In Kuhn HW and Tucker AW (eds.) Contributions to the Theory of Games II, Annals of Mathematics Studies No. 28, Princeton University Press, Princeton, New Jersey, pp. 307317

[5] Willson, SJ (1993) A Value for Partially Defined Cooperative Games. Int J Game Theory 21:371-384

[6] Young HP (1985) Monotonic Solutions of Cooperative Games. Int J Game Theory 14:65-72 